\title{
Minocycline and cisplatin exert synergistic growth suppression on hepatocellular carcinoma by inducing $S$ phase arrest and apoptosis
}

\author{
FU-YAO LIU ${ }^{1}$, YAN-HUI WU ${ }^{1}$, SHAO-JUN ZHOU ${ }^{1}$, YUE-LING DENG ${ }^{2}$, \\ ZUN-YI ZHANG ${ }^{1}$, ER-LEI ZHANG ${ }^{1}$ and ZHI-YONG HUANG ${ }^{1}$ \\ ${ }^{1}$ Research Laboratory and Hepatic Surgical Center, Department of Surgery, Tongji Hospital of
Tongji Medical College of Huazhong University of Science and Technology, Wuhan, Hubei;
${ }^{2}$ Department of Gastroenterology, Mianyang Center Hospital, Mianyang, Sichuan, P.R. China
}

Received April 1, 2014; Accepted May 6, 2014

DOI: $10.3892 /$ or.2014.3248

\begin{abstract}
Minocycline, a semisynthetic tetracycline, is a highly lipophilic molecule capable of infiltrating tissues and blood. Previous studies have revealed the functions and mechanisms of minocycline in anti-inflammation, protection of the nervous system and certain tumors. The role of minocycline has never been investigated in hepatocellular carcinoma (HCC). The functions of minocycline on HCC cells were investigated using immunohistochemical staining and western blotting. Minocycline was applied to L02, HepG2 and Huh7 cells, and the growth characteristics were studied. Cisplatin was administered in combination with minocycline in this study. Cell cycle and apoptosis analyses were employed to investigate the mechanisms underlying the growth regulation associated with minocycline and(or) cisplatin. Minocycline caused S phase cell cycle arrest and an increase in the apoptotic rate associated with upregulation of p27, cleaved-caspase8, cleaved-caspase 3 and cleavedPRAP-1. Low dose of cisplatin promoted cell cycle arrest and apoptosis, whereas minocycline was mainly associated with upregulation of cleaved-PARP-1. The combination of cisplatin and minocycline increased the rate and extent of cell cycle arrest and increased the apoptosis rate caused by minocycline. A novel mechanism was revealed. Minocycline functions as an antitumor drug in HCC by regulating p27, caspase-3 and PARP-1. Cisplatin enhanced minocycline's effect on PARP-1.
\end{abstract}

Correspondence to: Professor Zhi-Yong Huang, Research Laboratory and Hepatic Surgical Center, Department of Surgery, Tongji Hospital of Tongji Medical College of Huazhong University of Science and Technology, 1095 Jiefang Avenue, Wuhan, Hubei 430030, P.R. China E-mail: zyhuang@medmail.com.cn

Key words: HCC, minocycline, cisplatin, PARP-1, P27

\section{Introduction}

Hepatocellular carcinoma (HCC) is the fifth leading cause of all cancer-related deaths worldwide, and most of these deaths occur in developing countries (1). In recent years, studies regarding the pathogenesis of HCC, from microbial metabolites, a new type of oncogene to microRNAs (1-4), have weaved an intricate network. However, little progress in curing this cancer has been achieved. HCC is also characterized by its insensitivity to chemotherapy and radiotherapy. Although several targeted therapeutic drugs have been discovered (5), their clinical effects on HCC still require further observation. For example, sorafenib blocks the RAF/MEK/ERK pathway, inhibits tumor angiogenesis, and induces tumor cell apoptosis (6). Based on a previous study (7), PJ34, an inhibitor of PARP-1, a nuclear enzyme that not only responds to DNA damage and facilitates DNA repair, but also mediates cell death through a caspase-independent pathway (8), was found to effectively suppress proliferation of HepG2 cells. The inhibitory effect of minocycline at nanomolar concentrations on PARP-1 was revealed nearly 10 years ago (9).

Minocycline, a semisynthetic tetracycline, is a highly lipophilic molecule capable of infiltrating tissues and blood (10). As a replacement of earlier tetracycline, minocycline, which has a low propensity to produce antibiotic resistance, is commonly used to treat many types of infections. With further exploration of its functions and mechanisms, particular aspects of anti-inflammation (11) and protection of the nervous system (12-14), minocycline has found a wider and deeper utilization. Only recently has research examined how minocycline inhibits proliferation and promotes apoptosis (15). The dosage required to induce effective apoptosis was tens of folds higher than the dosage used for treatment of inflammation. Because of this, there is substantial toxicity associated with minocycline treatment (16). Therefore, the combination of chemotherapeutics with minocycline is a new method by which to combat the toxicity issues associated with high dosages of minocycline alone.

Cisplatin crosslinks DNA in several different ways, interfering with mitotic cell division. Cells undergo DNA damage 
responses that induce apoptosis when repair is not possible. Cisplatin elicits a complex response in the cell, including apoptosis, DNA repair and drug resistance involving the ATR, p53, p73 and MAPK pathways (17). The effects of cisplatin treatment on apoptosis and DNA repair are enhanced when used in combination with minocycline. Cisplatin treatment is also limited by toxicity $(18,19)$.

Herein, we present our in vitro and in vivo results to show that minocycline treatment induces cell cycle arrest and apoptosis. Additionally, when low-dose cisplatin was used in combination with minocycline, the suppression of HCC cell proliferation and apoptosis was enhanced above the level presented when using a single agent alone.

\section{Materials and methods}

Cell lines, cell culture and transient transfection. The human embryonic liver cell line L02 and HCC cell lines Huh7 and HepG2 were purchased from the Typical Training Content Preservation Committee Cell Bank, of the Chinese Academy of Sciences (China). The cells were cultured in Dulbecco's modified Eagle's medium (DMEM; Gibco-BRL, USA) supplemented with $10 \%$ fetal bovine serum (FBS) and $1 \%$ penicillin/streptomycin at $37^{\circ} \mathrm{C}$ in a humidified atmosphere with $5 \% \mathrm{CO}_{2}$.

p27 siRNAs (RiboBio Co. Ltd., China) were used to transiently transfect HepG2 cells. The transfection was performed using Lipofectamine ${ }^{\circledR} 2000$ (Invitrogen, Carlsbad, CA, USA), according to the manufacturer's instructions. Cells were seeded at a density of $5 \times 10^{4}$ per well in a 6 -well plate.

Cell proliferation assay. Cells were seeded in 6-cm plates at $5 \times 10^{4}$ cells per well. The cells were cultured with minocycline and/or cisplatin at different concentrations. Cell numbers were counted on days 3,6, and 9 after seeding. The assay was repeated three times.

Soft agar colony formation assay. A soft agar colony formation assay was performed for the transfected and control cells. Briefly, $1 \times 10^{3}$ cells were seeded into a 6 -well plate in a medium containing $0.3 \%$ noble agar and cultured for 14 days. The number of colonies was determined by direct counting using an inverted microscope (Nikon, Japan).

Flow cytometry to analyze the cell cycle and apoptosis. For cell cycle analysis, $1 \times 10^{5}$ HepG 2 cells were washed three times in PBS and serum starved for $48 \mathrm{~h}$. The cells were stimulated with DMEM containing $10 \%$ FBS for 24,48 , or 72 h. Cells $\left(1 \times 10^{4}\right)$ were analyzed from each sample on a FACSCalibur flow cytometer (Becton Dickinson).

For the apoptosis analysis, $1 \times 10^{5}$ cells per well were seeded in 6-well plates and incubated with DMEM containing $10 \%$ FBS. After $48 \mathrm{~h}$, cells were stained for $15 \mathrm{~min}$ with FITC-Annexin $\mathrm{V}$ and propidium iodide in binding buffer and then analyzed by flow cytometry within $1 \mathrm{~h}$.

Xenograft assay. Male Balb/c nude mice $(\mathrm{n}=18)$ were provided by the Experimental Animal Center of the Tongji Medical College. HepG2 cells $\left(2 \times 10^{6}\right)$ were injected into each of the bilateral flanks of the mice. On day 14 post injection, the mice were randomly divided into three groups: the minocycline, minocycline and cisplatin, and control groups.

Minocycline at $6 \mathrm{mg} / \mathrm{kg}$ in $0.1 \mathrm{ml}$ was injected intraperitoneally, and cisplatin was injected intraperitoneally at $0.3 \mathrm{mg} /$ $\mathrm{kg}$. The same volume of saline was injected into the control group. The tumors were measured every 3 days and tumor sizes were calculated on day 21 .

TUNEL assay. Sections of tumor tissues were deparaffinized in xylene, rehydrated, washed with PBS, and treated with $20 \mu \mathrm{g} / \mathrm{ml}$ of Proteinase $\mathrm{K}$ (Roche) for $10 \mathrm{~min}$ at room temperature. Then reactions were conducted using the TUNEL Apoptosis Assay $\mathrm{Kit}^{\circledR}$ (Roche) according to the manufacturer's instructions, and detected using DAB. The percentage of TUNEL-positive cells was assessed in five randomly selected fields for each tissue section.

Western blotting. Western blotting was performed with specific primary antibodies against CDK2, CDK4, p53, PARP-1, p21, caspase-3, casapase-8, ku80 and p27 (rabbit anti-human antibody; 1:1000; Epitomics, Burlingame, CA, USA), followed by the appropriate secondary HRP-conjugated antibodies (1:5000; Pierce, Rockford, IL, USA). The protein bands were visualized using an enhanced chemiluminescence detection system (Pierce).

Statistical analysis. ANOVA was performed to determine the statistical significance among the groups. A P-value $<0.05$ was considered to indicate a statistically significant result. All experimental data were analyzed using the SPSS statistical software (version 16.0).

\section{Results}

Minocycline inhibits the proliferation of HCC cell lines, but not the normal liver cell line. When HepG2 or Huh7 cells were cultured with minocycline at concentrations of 50, 75, and $100 \mu \mathrm{M}$, cell proliferation was significantly suppressed compared to the control cells cultured without minocycline (Fig. 1A). The suppressive effects of minocycline were dose-dependent, and the number of cells in the treated group was significantly lower than the number of cells in the control group on days 6 and 9. However, when the normal human liver cell line L02 was cultured with minocycline at $100 \mu \mathrm{M}$, no obvious suppressive effect was observed on days 3, 6 and 9 (Fig. 1D). At a concentration of $200 \mu \mathrm{M}$, minocycline strongly suppressed the proliferation of HCC cells, but it also weakly suppressed L02 cell proliferation. Furthermore, the soft agar assay revealed that minocycline treatment significantly inhibited colony formation by $50.5-64.5 \%$ compared to the control clones (Fig. 1B).

Inhibition of HCC cell proliferation by minocycline is enhanced by low-dose cisplatin. When HepG2 cells were cultured with minocycline at a concentration of $100 \mu \mathrm{M}$ in combination with $2 \mu \mathrm{M}$ or $5 \mu \mathrm{M}$ cisplatin, the level of suppression of cell proliferation was comparable to that of treatment with $200 \mu \mathrm{M}$ minocycline. The suppression was greater than with treatment of $2 \mu \mathrm{M}$ or $5 \mu \mathrm{M}$ cisplatin alone (Fig. 1C). There was no effect on L02 cells. Although treatment of HepG2 cells 

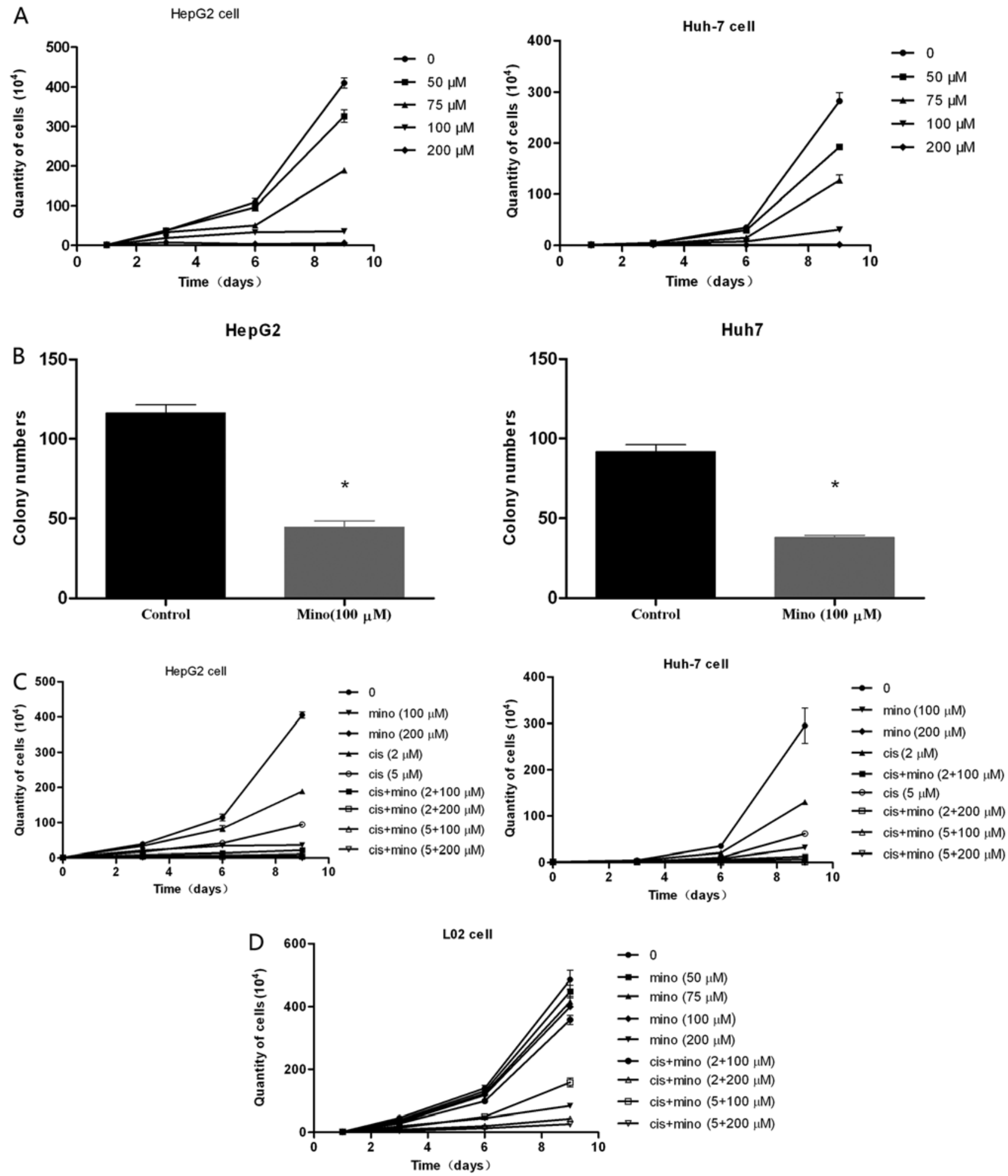

Figure 1. Minocycline inhibits the proliferation of HCC cells, but not normal liver cells. (A) As the concentration increased, minocycline gradually inhibited the proliferation of HepG2 and Huh7 cells, but had no apparent effect on L02 cells. When the concentration reached $200 \mu \mathrm{M}$, minocycline totally inhibited the proliferation of these cells. (B) Soft agar colony formation assay showed the obvious arrest of proliferation of HepG2 and Huh7 cells by minocycline compared with the control cells. (C) When $2 \mu \mathrm{M}$ cisplatin was applied with $100 \mu \mathrm{M}$ minocycline, an effect similar to $200 \mu \mathrm{M}$ minocycline was noted. (D) Minocycline had no obvious effect on the proliferation of L02 cells until its concentration reached $200 \mu \mathrm{M}$. The combination of $2 \mu \mathrm{M}$ cisplatin and $100 \mu \mathrm{M}$ minocycline had no obvious suppressive effect on L02 proliferation. ${ }^{*} \mathrm{P}<0.05 ;{ }^{* *} \mathrm{P}<0.01$. Cis, cisplatin; mino, minocycline.

with a concentration of $200 \mu \mathrm{M}$ minocycline in combination with $2 \mu \mathrm{M}$ or $5 \mu \mathrm{M}$ cisplatin decreased proliferation even further, this concentration also suppressed the proliferation of L02 cells.
Cisplatin enhances the effects of minocycline in regards to increased cell apoptosis and cell cycle arrest. HCC cells were divided into four treatment groups: the control; $100 \mu \mathrm{M}$ minocycline; $2 \mu \mathrm{M}$ cisplatin; and $100 \mu \mathrm{M}$ minocycline and $2 \mu \mathrm{M}$ 

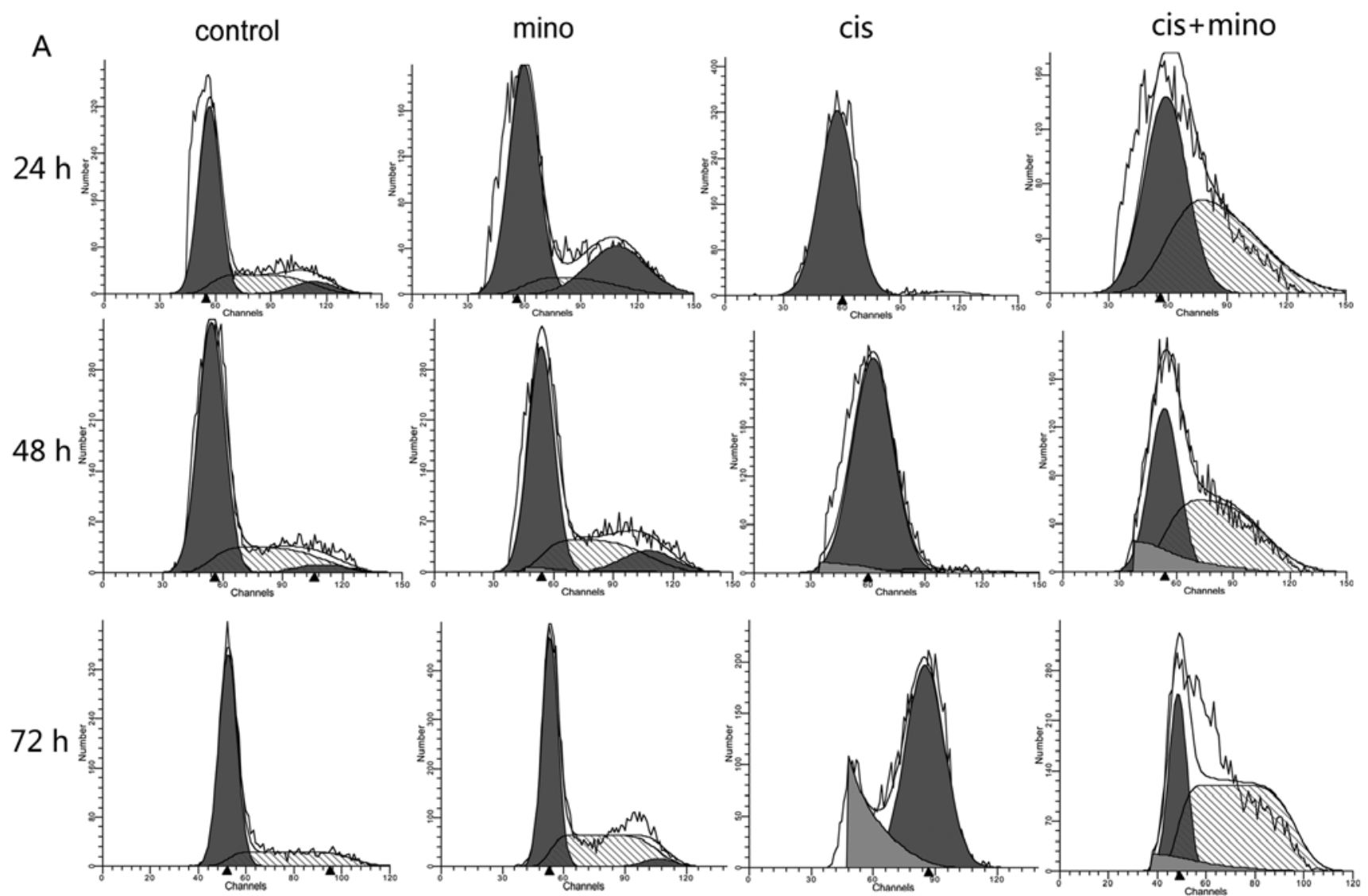

B
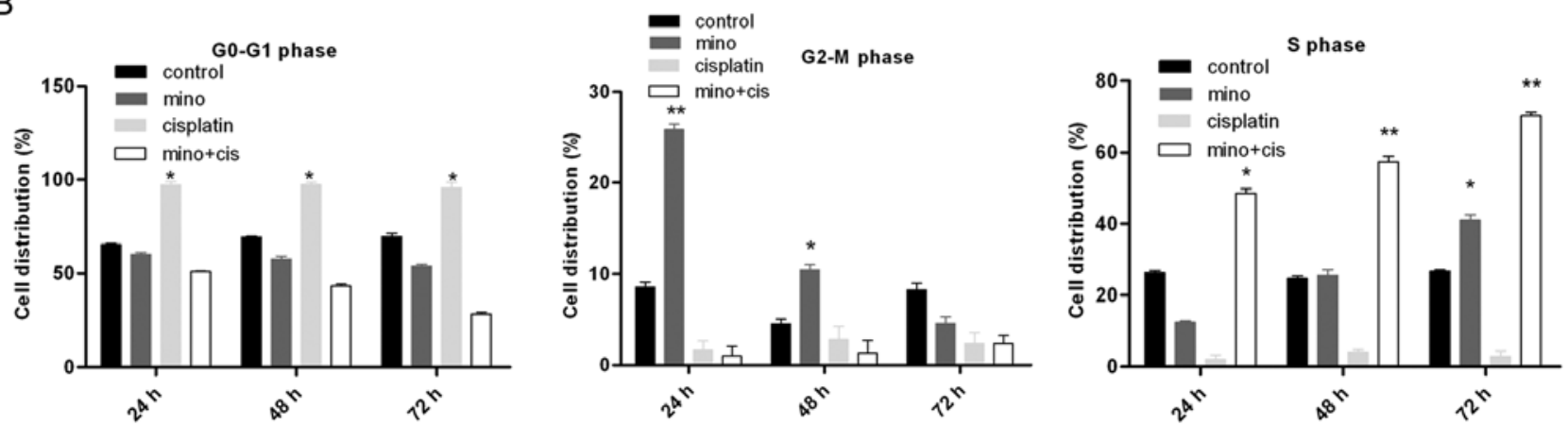

Figure 2. Low doses of cisplatin plus minocycline accelerate and induce almost total S phase arrest in HCC cells. (A and B) In the flow cytometric experiment, with time, minocycline induced the S phase arrest of HCC cells gradually, particularly at $72 \mathrm{~h}$ (cell distribution at S phase was $>40 \%$ ); although cisplatin induced nearly total G0-G1 phase arrest. When $2 \mu \mathrm{M}$ cisplatin was added to $100 \mu \mathrm{M}$ minocycline, it induced the obvious S phase arrest at $24 \mathrm{~h}$ and the effect was strengthened with time. ${ }^{*} \mathrm{P}<0.05 ;{ }^{* *} \mathrm{P}<0.01$. Cis, cisplatin; mino, minocycline.

cisplatin groups. At $24 \mathrm{~h}$, minocycline had no effect on cell cycle arrest. However, at 48 and $72 \mathrm{~h}$, more cells were arrested in the S phase, with distribution rates of $49.1 \pm 0.54,57.6 \pm 0.39$ and $71.2 \pm 1.2 \%$ at the three time points, respectively. Moreover, low-dose cisplatin induced G0/G1 cell cycle arrest in almost all cells. The combination of minocycline and cisplatin significantly accelerated the $\mathrm{S}$ phase arrest at all three time points (Fig. 2A and B). Minocycline treatment alone only induced S phase arrest after $72 \mathrm{~h}(40 \pm 0.94 \%)$, while the combination of minocycline and cisplatin induced $\mathrm{S}$ phase arrest after $24 \mathrm{~h}(49.1 \pm 0.54 \%)$. After $72 \mathrm{~h}$, the combination treatment induced $S$ phase arrest at a rate of $71.2 \pm 1.2 \%$. Additionally, the G0/G1 phase arrest induced by cisplatin treatment was eliminated with combination therapy, with rates decreasing from $97.6 \pm 1.38$ to $4.1 \pm 1.22 \%$.
Flow cytometry was used to analyze the induction of apoptosis in these cells. The apoptotic rates in the control, $100 \mu \mathrm{M}$ minocycline, $200 \mu \mathrm{M}$ minocycline, $2 \mu \mathrm{M}$ cisplatin, $100 \mu \mathrm{M}$ minocycline and $2 \mu \mathrm{M}$ cisplatin, and $200 \mu \mathrm{M}$ minocycline and $2 \mu \mathrm{M}$ cisplatin groups were $5.81 \pm 0.15,43.83 \pm 0.25,51.44 \pm 1.52$, $20.31 \pm 0.44,60.26 \pm 1.72$ and $67.34 \%$, respectively (Fig. 3A and B). Late stage apoptotic rates in the groups treated with $100 \mu \mathrm{M}$ minocycline, $200 \mu \mathrm{M}$ minocycline, or $2 \mu \mathrm{M}$ cisplatin were much higher than the rate in the control group. In the group treated with $200 \mu \mathrm{M}$ minocycline, the apoptotic rate reached $51.44 \pm 1.52 \%$, but in the group that was treated with the combination of $100 \mu \mathrm{M}$ minocycline and $2 \mu \mathrm{M}$ cisplatin, the apoptotic rate reached $60.26 \pm 1.72 \%$. The group that was treated with combination therapy also had a significantly higher rate of apoptosis than the control group. 

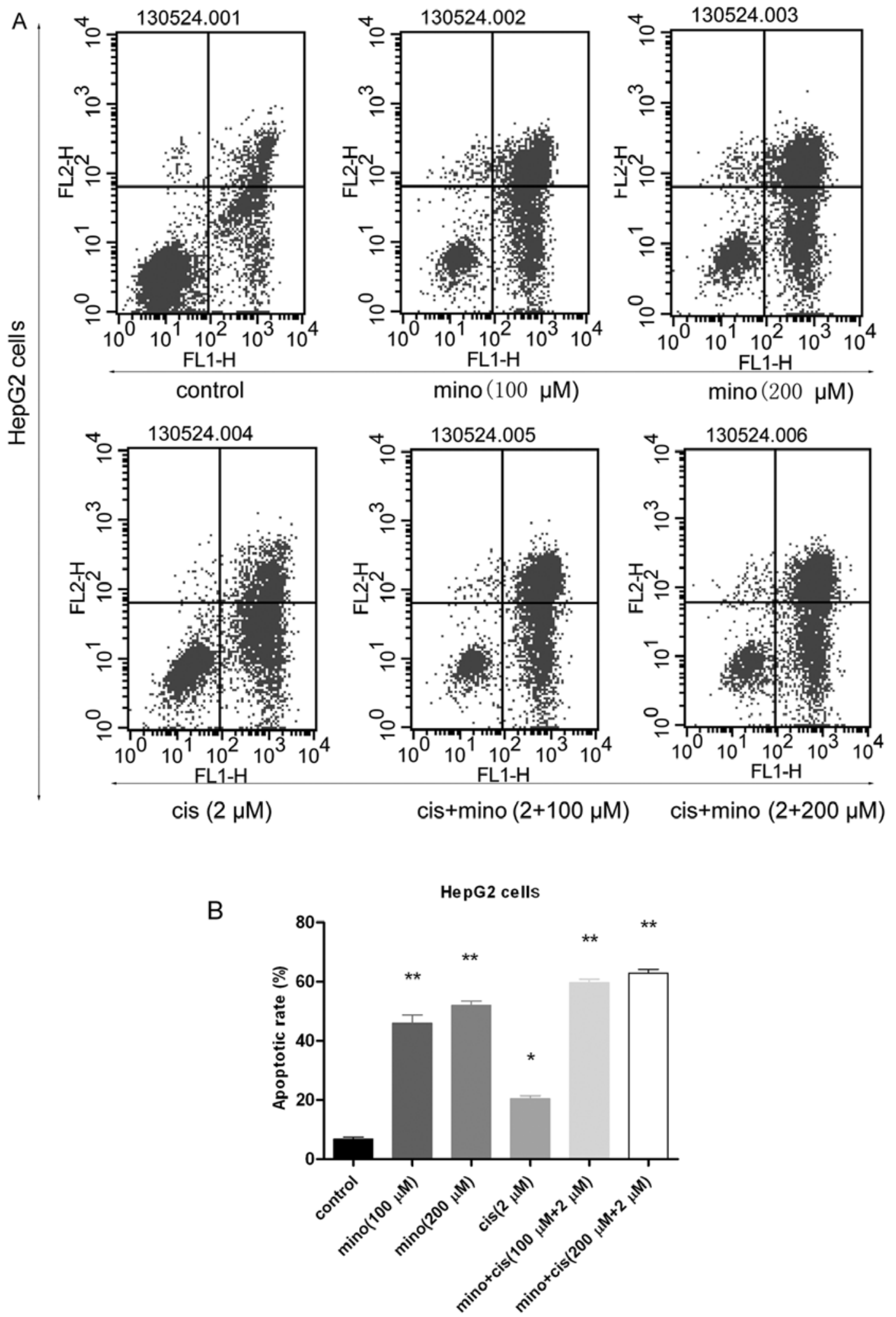

Figure 3. Low dose of cispaltin enhances the apoptosis of HCC cells induced by minocycline. (A and B) In the flow cytometric experiment, minocycline induced apoptosis of HCC cells ( $>40 \%$ at $100 \mu \mathrm{M}$ and $>50 \%$ at $200 \mu \mathrm{M}$ ). Combined with a low dose of $2 \mu \mathrm{M}$ cisplatin, the apoptotic rate reached nearly $60 \%$. ${ }^{*} \mathrm{P}<0.05 ;{ }^{* *} \mathrm{P}<0.01$. Cis, cisplatin; mino, minocycline.

Minocycline induces apoptosis in HCC cells through a mitochondrial-independent pathway and by suppressing the DNA repair process. Cleaved caspase- 3 was upregulated in HCC cells treated with minocycline. Since cleaved caspase- 8 was also upregulated in HCC cells treated with minocycline, the extrinsic apoptosis pathway was activated. The downregulation of PARP-1 elucidated another pathway associated with apoptosis induced by minocycline. In addition to PARP-1, the 

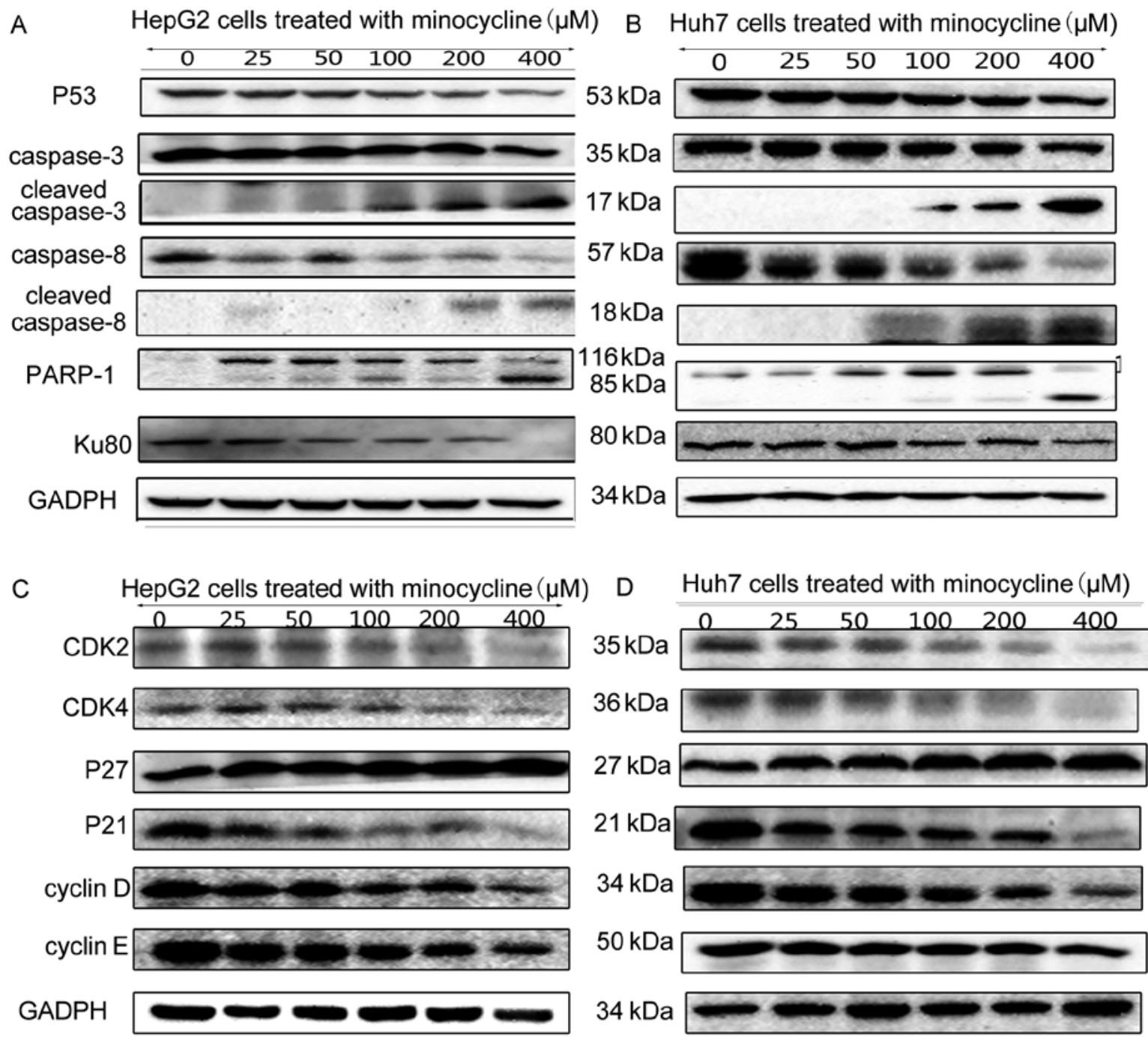

Figure 4. Minocycline induces apoptosis and cell cycle arrest by regulating PARP-1, p27 and extrinsic apoptotic factors. (A and B) Minocycline suppressed the expression of DNA repairing protein PARP-1 and Ku80. Minocycline also regulated the expression of caspase-3 and caspase-8, the important factors of the extrinsic apoptotic pathway. (C and D) Minocycline downregulated the expression of CDK2 and CDK4 complexes by suppressing p27.

expression of another DNA repair factor, Ku80, was suppressed by minocycline treatment (Fig. 4A and B).

Minocycline induces HCC cell cycle arrest through the p27 pathway. In HepG2 cells, the expression levels of CDK2, $\mathrm{CDK} 4$, cyclin $\mathrm{D}$, and cyclin $\mathrm{E}$ were significantly decreased in cells treated with minocycline (Fig. 4C and D), while minocycline treatment had no effect on the expression of p53 and p21. However, the expression of p27 was dose-dependently upregulated with minocycline treatment (Fig. 4C and D). To verify this result, p27 expression was knocked down by siRNA, When the p27 expression was blocked, the minocycline-induced suppression of downstream factors, such as CDK2, was reversed (Fig. 5A).

Cell proliferation assay demonstrated that the proliferation of the HepG2 cells treated with minocycline was significantly increased after transfection with p27-siRNA compared with the mock-transfected cells or the vector-transfected control cells on days 6 and 9 of culture (Fig. 5B). There was no significant difference in cell numbers between the mock- , vector- and
p27-siRNA-transfected cells which were not treated by minocycline at all time points after transfection.

Cisplatin enhances the effect of minocycline on cell cycle arrest and apoptosis. Cisplatin can induce apoptosis through both the extrinsic and intrinsic pathways. Cisplatin can also increase the expression of p53, which activates p21 to induce cell cycle arrest in the G0/G1 phase, and it also can downregulate the expression of PARP-1 and suppress the expression of p27. In vitro assays showed that the combination of cisplatin and minocycline enhanced the levels of apoptosis in HCC cells when compared with the application of a single drug. The expression of cleaved caspase- 3 and p53 was upregulated following treatment with the combination therapy when compared to that induced by treatment with minocycline alone (Fig. 6A and B). Although CDK2 and CDK4 expression levels were decreased, that was not enough to induce the suppressive effect on HCC cells observed at early time points. Therefore, we hypothesized that the $\mathrm{S}$ phase arrest induced by the combination of the two drugs was caused mainly by suppression 
A

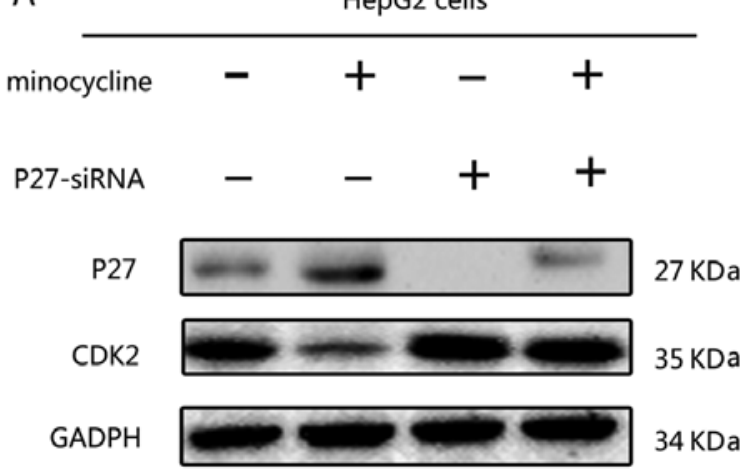

B

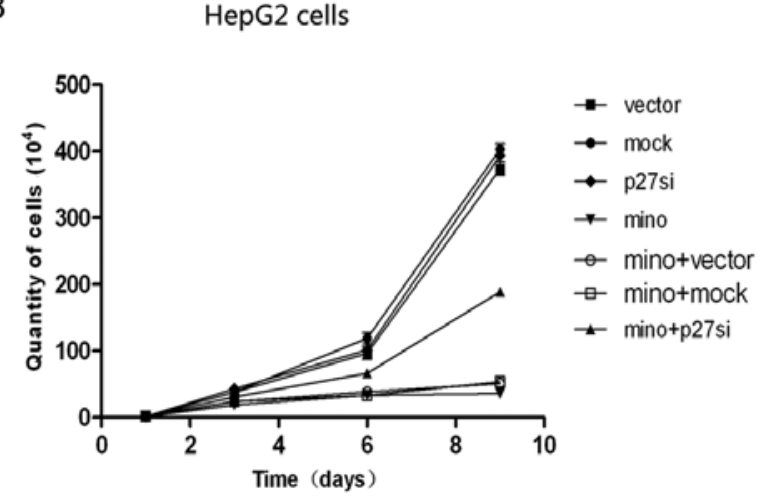

Figure 5. Minocycline-induced growth suppression and cell cycle arrest are dependent on the p27 pathway. (A) Blockage of p27 recovered the suppression of CDK2 induced by minocycline thus showing that 27 is the direct target of minocycline. (B) Blockage of p27 partly recovered the suppression of the proliferation induced by minocycline. Mino, minocycline.

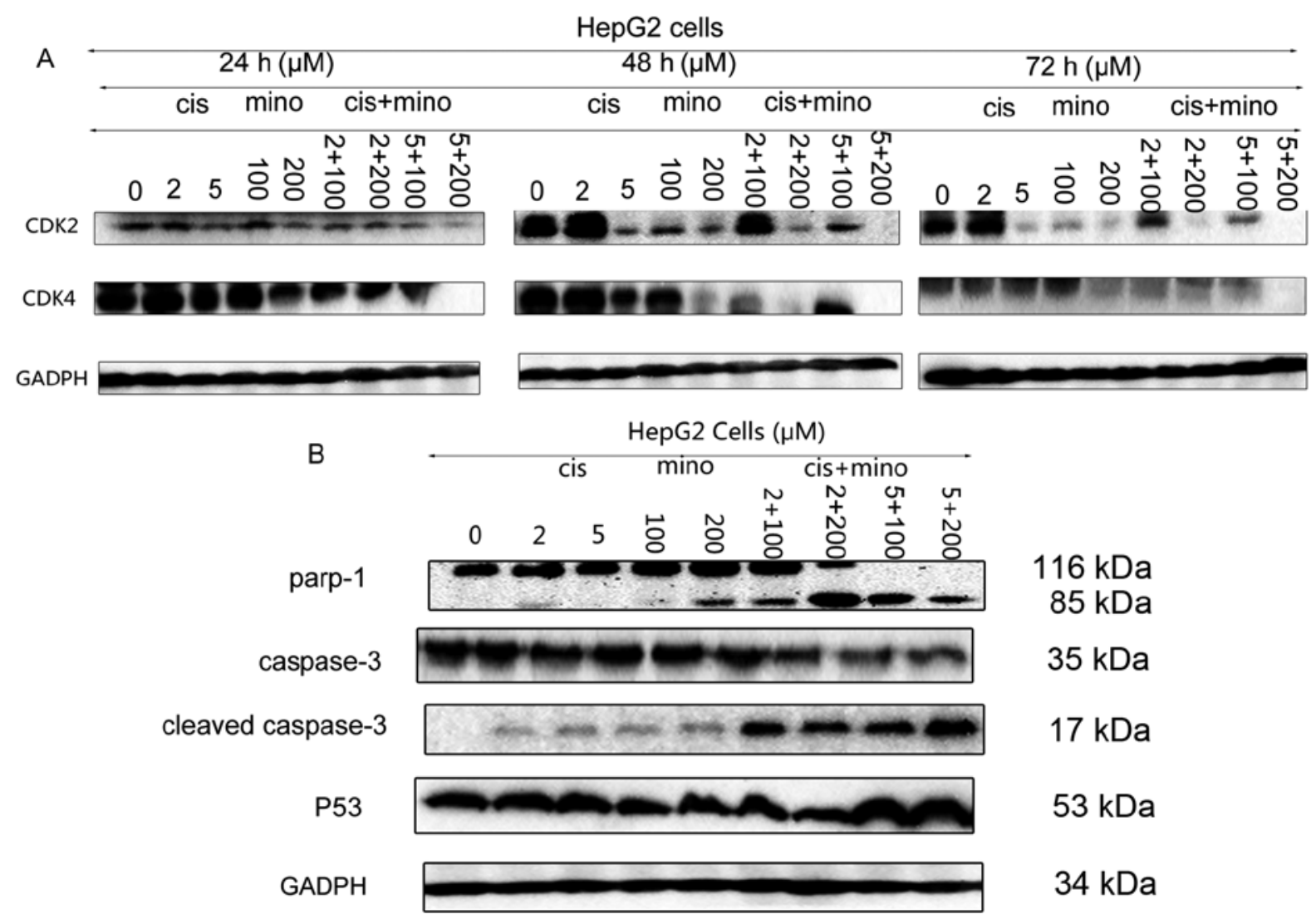

Figure 6. The combined application of minocycline and cisplatin enhances the apoptosis and cell cycle arrest of HCC cells by regulating PARP-1, caspase-3, Ku80 and P53. (A) In the western blot assay, the combined application of minocycline and cisplatin obviously suppressed the expression of CDK2 and CDK4 compared with the application of minocycline or cisplatin alone. (B) The combined application of minocycline and cisplatin not only downregulated the expression of PARP-1, Ku80, caspase-3 and caspase-8 induced by minocycline, but also upregulated the expression of P53 compared with application of minocycline or cisplatin alone. Cis, cisplatin; mino, minocycline.

of the PARP-1 DNA repair pathway. The downregulation of PARP-1 verified our deduction (Fig. 6A and B).

Minocycline and cisplatin treatment inhibits HepG2 tumor growth in nude mice. Nude mice received injections of $2 \times 10^{6}$ HepG2 cells in each flank. Tumors formed after two weeks, and tumor-bearing mice were randomly divided into three groups. As shown in Fig. 7A, the mean volumes of the tumors from mice treated with minocycline $\left(0.70 \mathrm{~cm}^{3}\right)$ or cisplatin in combination with minocycline $\left(0.45 \mathrm{~cm}^{3}\right)$ were significantly smaller than those from the control group $\left(1.66 \mathrm{~cm}^{3}\right)$, and tumors from mice treated with the combination therapy were significantly smaller than tumors from mice treated with minocycline alone. In addition, when compared to tumors from the control group, tumors from mice that received any treatment grew at significantly slower rates and were smaller at 
A

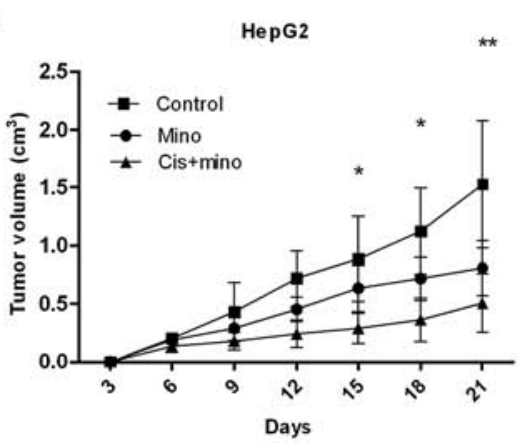

C

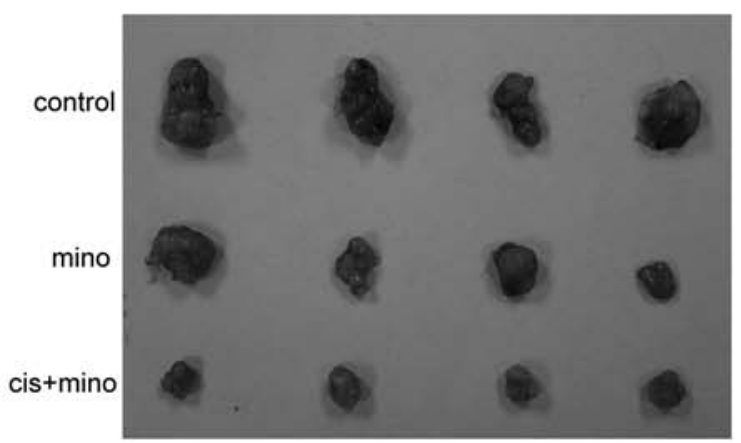

B

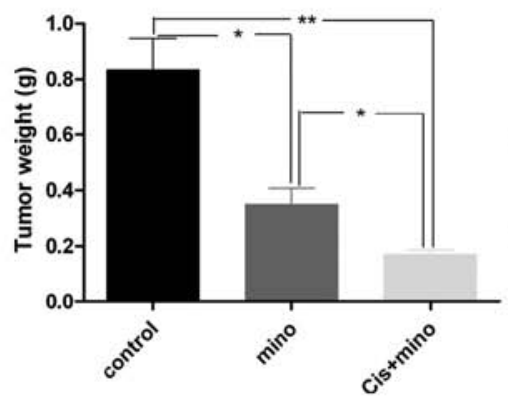

E

$400 x$
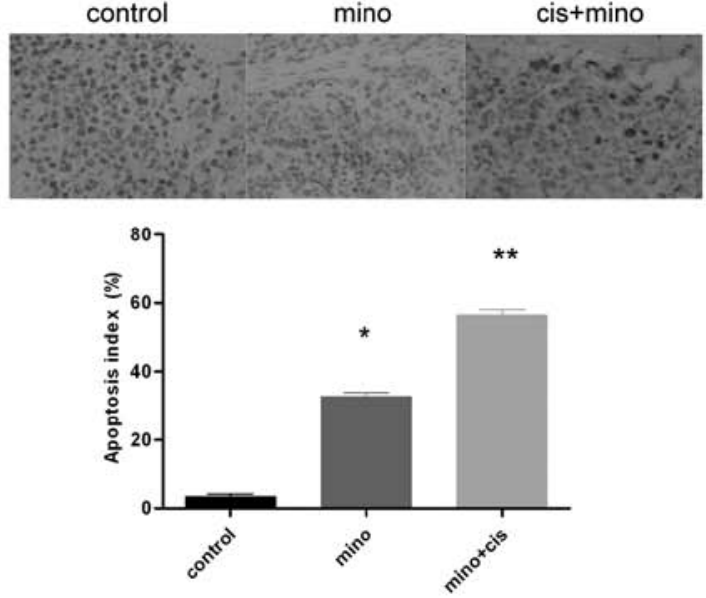

Figure 7. Minocycline decreases the volume and weight of nude mouse transplantation tumors, and this effect is enhanced by cisplatin. (A-C) The weight and volume of nude mouse transplantated tumors were significantly different among the control, minocycline and cisplatin + minocycline groups. (D) As determined by western blot assay in nude mouse transplantation tumors, the combined application of minocycline and cisplatin downregulated the expression of PARP-1, Ku80, caspase-3 and upregulated the expression of P53. (E) Apoptosis assessed by the TUNEL assay. Minocycline and/or cisplatin induced apoptosis of tumor cells compared with the control group. ${ }^{*} \mathrm{P}<0.05 ;{ }^{* *} \mathrm{P}<0.01$. Cis, cisplatin; mino, minocycline.

all time points examined from day 24 post-injection (Fig. 7A). The mass of tumors from mice that received minocycline alone or combination therapy was significantly lower than that of tumors from mice in the control group (Fig. 7B and C), and the mass of tumors from the mice that received combination therapy was significantly smaller than that from tumors from the mice treated with minocycline alone. Western blot analysis (Fig. 7D) verified the effect of minocycline and/or cisplatin on the expression of PARP-1, caspase- 3 , and Ku80 in vitro. As shown by TUNEL assay, the rate of apoptosis was calculated. As shown in Fig. 7E, treatment with minocycline alone or minocycline in combination with cisplatin resulted in a significantly higher apoptotic index (32.7 and 56.4\% respectively), when compared to the control group.

\section{Discussion}

Minocycline is a second-generation tetracycline antibiotic with favorable pharmacological properties. It is also used as an anti-inflammatory and neuroprotective agent. In this report, we present novel and direct evidence that minocycline markedly induces apoptosis and cell cycle arrest in liver cancer cells.

Minocycline inhibits PARP-1 expression at nanomolar concentrations (9), and this research suggests that minocycline could be useful to treat HCC. Based on a previous study (7), an inhibitor of PARP-1 suppressed the proliferation of HCC cells. We hypothesized that minocycline could function similarly, and should be investigated further given its ability to infiltrate tissues and blood. We treated several HCC cell lines with minocycline at different concentrations. We found that the proliferation of liver cells was suppressed when the suitable dosage of minocycline was applied. However, minocycline had no suppressive effect on the proliferation of L02 cells at the same dosage. Moreover, minocycline treatment changed the morphology of HepG2 and Huh7 cells from smooth and plump to shrinking. The cell nucleus also appeared to undergo distinctive changes, including pyknosis and karyolitic and apoptotic body formation, a typical feature of apoptosis (20). Using flow cytometry, we determined that minocycline treatment induced $\mathrm{S}$ phase arrest with an increase in concentration, especially after $72 \mathrm{~h}$. Moreover the late stage apoptotic cells increased with its concentration.

Western blot analysis was used to explore the underlying mechanism responsible for this phenomenon. Treatment with increasing concentrations of minocycline increased the expression levels of CDK2, CDK4, cyclin D, and cyclin E, which explains the observed cell cycle arrest noted with treatment (21). We then examined the expression of the upstream factors of CDK2 and CDK4: p53, p21 and p27. Minocycline treatment had no effect on the expression of $\mathrm{p} 53$ or $\mathrm{p} 21$, the downstream factor of p53 (22). However, p27, which also 
regulates CDKs and cyclins $(23,24)$, was induced following minocycline treatment, and the suppression of CDKs and cyclins by minocycline was reversed by blocking p27 expression. We, therefore, concluded that minocycline regulates cell cycle arrest by activating the p 27 pathway, but the mechanism by which minocycline induced apoptosis of HCC cell lines remain unclear.

We then tested the effects of minocycline treatment on other apoptotic factors induced by mitochondria $(25,26)$, Minocycline did not affect the intrinsic mitochondrial apoptotic pathway. Minocycline treatment induced both the expression of cleaved caspase- 3 and cleaved caspase- 8 , which indicated the activation of the extrinsic apoptotic pathway $(27,28)$.

A decrease in PARP-1 expression, as noted with minocycline treatment, has been shown to affect both cell cycle arrest and apoptosis in other pathways $(8,29,30)$. Through suppressed PARP-1, minocycline induced HCC cells into apoptosis directly.

Although minocycline induces apoptosis and cell cycle arrest, the high doses needed also have toxicity to normal cells (16). Therefore, we wanted to use another drug in combination with minocycline so that we could reduce the dosage but still have a similar effect on cancer cell death. Cisplatin can induce apoptosis through both the extrinsic and intrinsic apoptotic pathways $(31,32)$, which has some overlap with the functions of minocycline. Then we chose it to continue our research. The combination of cisplatin and minocycline enhanced the apoptosis of HCC cells when compared with treatment with either minocycline or cisplatin alone. The expression of p53 was also increased, suggesting that the combination of these two drugs may have synergy in inducing apoptosis.

Based on the findings of other studies, cisplatin induced the expression of p21 while decreasing p27 expression (33), thereby antagonizing the suppressive effects of minocycline on CDK2 and CDK4 complexes. Cisplatin treatment induced G0/G1 arrest, but the combination of minocycline and cisplatin induced $\mathrm{S}$ phase arrest. Minocycline induced $\mathrm{S}$ phase arrest after $72 \mathrm{~h}$, thus we concluded that p27 upregulation was not the primary mechanism. The strong suppressive effect of this combination on PARP-1 expression blocked the repair of HCC cells and inhibited damaged cells from entering the $\mathrm{G} 2 / \mathrm{M}$ phase, thus inducing cell accumulation in the $\mathrm{S}$ phase and apoptosis. This synergy of drugs was enhanced over time. In the animal model, subcutaneous tumors were implanted in Balb/c nude mice. The tumors in the group treated with the combination therapy were much smaller than the tumors in both groups treated with a single drug and the control group, by volume and weight. Western blotting and TUNEL assay of tumors showed the same effect on apoptosis and proliferation with in vitro experiments.

Then KLF17, one of the typical factors associated with HCC metastasis (34), was tested by western blotting. Minocycline also upregulated its expression (data not shown). Minocycline also had some effect on inhibiting HCC metastasis.

In conclusion, minocycline inhibited $\mathrm{HCC}$ cell proliferation by inducing apoptosis and cell cycle arrest through extrinsic apoptosis, P27 and PARP-1. In addition, a low dose of cisplatin enhanced minocycline apoptosis capacity. In addition, cisplatin enhanced $\mathrm{S}$ phase arrest induced by minocycline through suppressed PARP-1. Finally, cisplatin upregulated the expression of P53 which was not affected by minocycline. These data need further evaluation in clinical conditions and in other types of cancer, particularly in cancers that have a tight relation with PARP-1 expression.

\section{References}

1. Jemal A, Bray F, Center MM, Ferlay J, Ward E and Forman D: Global cancer statistics. CA Cancer J Clin 61: 69-90, 2011.

2. Yoshimoto S, Loo TM, Atarashi K, et al: Obesity-induced gut microbial metabolite promotes liver cancer through senescence secretome. Nature 499: 97-101, 2013.

3. Min L, Ji Y, Bakiri L, et al: Liver cancer initiation is controlled by AP-1 through SIRT6-dependent inhibition of survivin. Nat Cell Biol 14: 1203-1211, 2012.

4. Xu X, Fan Z, Kang L, et al: Hepatitis B virus X protein represses miRNA-148a to enhance tumorigenesis. J Clin Invest 123: 630-645, 2013.

5. Maluccio M and Covey A: Recent progress in understanding, diagnosing, and treating hepatocellular carcinoma. CA Cancer J Clin 62: 394-399, 2012.

6. Liu L, Cao Y, Chen C, et al: Sorafenib blocks the RAF/MEK/ ERK pathway, inhibits tumor angiogenesis, and induces tumor cell apoptosis in hepatocellular carcinoma model PLC/PRF/5. Cancer Res 66: 11851-11858, 2006.

7. Huang SH, Xiong M, Chen XP, Xiao ZY, Zhao YF and Huang ZY: PJ34, an inhibitor of PARP-1, suppresses cell growth and enhances the suppressive effects of cisplatin in liver cancer cells. Oncol Rep 20: 567-572, 2008.

8. Yu S-W, Wang H,Poitras MF, et al: Mediation of poly(ADP-ribose) polymerase-1-dependent cell death by apoptosis-inducing factor. Science 297: 259-263, 2002.

9. Alano CC, Kauppinen TM, Valls AV and Swanson RA: Minocycline inhibits poly(ADP-ribose) polymerase-1 at nanomolar concentrations. Proc Natl Acad Sci USA 103: 9685-9690, 2006.

10. Yong VW, Wells J, Giuliani F, Casha S, Power C and Metz LM: The promise of minocycline in neurology. Lancet Neurol 3: 744-751, 2004

11. Le $\mathrm{CH}$, Morales A and Trentham DE: Minocycline in early diffuse scleroderma. Lancet 352: 1755-1756, 1998.

12. Zhu S, Stavrovskaya IG, Drozda M, et al: Minocycline inhibits cytochrome c release and delays progression of amyotrophic lateral sclerosis in mice. Nature 417: 74-78, 2002.

13. Zink MC, Uhrlaub J, DeWitt J, et al: Neuroprotective and anti-human immunodeficiency virus activity of minocycline. JAMA 293: 2003-2011, 2005.

14. Stefanova N, Bücke P, Duerr S and Wenning GK: Multiple system atrophy: an update. Lancet Neurol 8: 1172-1178, 2009

15. Pourgholami MH, Ataie-Kachoie P, Badar S and Morris DL: Minocycline inhibits malignant ascites of ovarian cancer through targeting multiple signaling pathways. Gynecol Oncol 129: 113-119, 2013.

16. Bouwman P and Jonkers J: The effects of deregulated DNA damage signalling on cancer chemotherapy response and resistance. Nat Rev Cancer 12: 587-598, 2012.

17. Siddik ZH: Cisplatin: mode of cytotoxic action and molecular basis of resistance. Oncogene 22: 7265-7279, 2003.

18. Fuertes MA, Alonso C and Pérez JM: Biochemical modulation of Cisplatin mechanisms of action: enhancement of antitumor activity and circumvention of drug resistance. Chemical Rev 103: 645-662, 2003.

19. Wang D and Lippard SJ: Cellular processing of platinum anticancer drugs. Nat Rev Drug Discov 4: 307-320, 2005.

20. Haubrich WS: Apoptosis. Gastroenterology 119: 1805, 2000.

21. Lord CJ and Ashworth A: The DNA damage response and cancer therapy. Nature 481: 287-294, 2012.

22. Abbas T and Dutta A: p21 in cancer: intricate networks and multiple activities. Nat Rev Cancer 9: 400-414, 2009.

23. Nakayama K, Nagahama H, Minamishima YA, et al: Targeted disruption of Skp2 results in accumulation of cyclin E and p27(Kip1), polyploidy and centrosome overduplication. EMBO J 19: 2069-2081, 2000.

24. le Sage C, Nagel R, Egan DA, et al: Regulation of the p27(Kip1) tumor suppressor by miR-221 and miR-222 promotes cancer cell proliferation. EMBO J 26: 3699-3708, 2007. 
25. PenningerJMand KroemerG: Mitochondria,AIFandcaspasesrivaling for cell death execution. Nat Cell Biol 5: 97-99, 2003.

26. Virag L, Robaszkiewicz A, Vargas JM and Oliver FJ: Poly(ADP-ribose) signaling in cell death. Mol Aspects Med 34 1153-1167 2013

27. Günther C, Martini E, Wittkopf N, et al: Caspase-8 regulates TNF-alpha-induced epithelial necroptosis and terminal ileitis. Nature 477: 335-339, 2011.

28. Jin Z, Li Y, Pitti R, et al: Cullin3-based polyubiquitination and p62-dependent aggregation of caspase- 8 mediate extrinsic apoptosis signaling. Cell 137: 721-735, 2009.

29. Stilmann M, Hinz M, Arslan SÇ, Zimmer A, Schreiber V and Scheidereit C: A nuclear poly(ADP-Ribose)-dependent signalosome confers DNA damage-induced IkappaB kinase activation. Mol Cell 36: 365-378, 2009.

30. Midorikawa R, Takei Y and Hirokawa N: KIF4 motor regulates activity-dependent neuronal survival by suppressing PARP-1 enzymatic activity. Cell 125: 371-383, 2006.
31. Vondálová Blanárová O, Jelinková I, Szöor A, et al: Cisplatin and a potent platinum(IV) complex-mediated enhancement of TRAIL-induced cancer cells killing is associated with modulation of upstream events in the extrinsic apoptotic pathway. Carcinogenesis 32: 42-51, 2011.

32. Barton C, Davies D, Balkwill F and Burke F: Involvement of both intrinsic and extrinsic pathways in IFN-gamma-induced apoptosis that are enhanced with cisplatin. Eur J Cancer 41: 1474-1486, 2005

33. Qin LF and Ng IO: Induction of apoptosis by cisplatin and its effect on cell cycle-related proteins and cell cycle changes in hepatoma cells. Cancer Lett 175: 27-38, 2002.

34. Liu FY, Deng YL, Li Y, et al: Down-regulated KLF17 expression is associated with tumor invasion and poor prognosis in hepatocellular carcinoma. Med Oncol 30: 425, 2013 